\title{
Performance analysis of power beacon-assisted D2D communication networks in the presence of eavesdropper and co-channel interference
}

\author{
Bui Vu Minh', and Van-Duc Phan² \\ ${ }^{1}$ Faculty of Mechanical, Electrical, Electronic and Automotive Engineering, Nguyen Tat Thanh University, \\ Ho Chi Minh City, Vietnam \\ ${ }^{2}$ Faculty of Automobile Technology, Van Lang University, Ho Chi Minh City, Vietnam
}

\section{Article Info}

Article history:

Received Jan 1, 2021

Revised Aug 26, 2021

Accepted Oct 12, 2021

\section{Keywords:}

Energy harvesting

Half-duplex

Monte carlo simulation

Non-zero secrecy probability

Relaying network

\begin{abstract}
Radiofrequency (RF) signals can provide both information and energy, which have excellent advantages (small dimensions, low cost, and independence concerning time and location in urban areas), can be considered as electrical sources for cooperative network devices. Performance analysis of power beacon-assisted D2D Communication Networks in the Presence of Eavesdropper and Co-channel Interference is presented is investigated. The outage probability and the intercept probability of the proposed system are analyzed and derived. The impact of the main system parameters on the system performance is investigated. The monte carlo simulation is used for verifying the correctness of the analytical section in this paper.
\end{abstract}

This is an open access article under the CC BY-SA license.

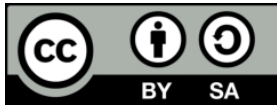

Corresponding Author:

Van-Duc Phan

Faculty of Automobile Technology

Van Lang University

Ho Chi Minh City, Vietnam

Email: duc.pv@vlu.edu.vn

\section{INTRODUCTION}

Radiofrequency (RF) signals can provide both information and energy, which have excellent advantages (small dimensions, low cost, and independence concerning time and location in urban areas), can be considered as electrical sources for cooperative network devices [1]-[10]. In recent years, harvesting energy from radio frequency (RF) signals has drawn significant research interest as a promising solution to solve the energy problem. This energy collection method, referred to as RF energy harvesting, has clear advantages over other energy harvesting techniques due to its predictable, controllable, and stable nature. The research in RF energy harvesting mainly falls into two broad categories: Simultaneous wireless information and power transfer (SWIPT) and wireless powered communication network (WPCN) [10]-[18].

The main contributions of this paper are:

- Performance analysis of power beacon-assisted D2D communication networks in the presence of eavesdropper and co-channel interference is presented is investigated.

- $\quad$ The outage probability and the intercept probability of the proposed system are analyzed and derived.

- $\quad$ The impact of the main system parameters on the system performance is investigated.

- The monte carlo simulation is used for verifying the correctness of the analytical section. 


\section{SYSTEM MODEL}

In this research, the proposed system model with the energy harvesting (EH) and information processing (IT) is illustrated as shown in [19]-[25]. The proposed system model is drawn in Figure 1 and the time switching protocol is presented in Figure 2.

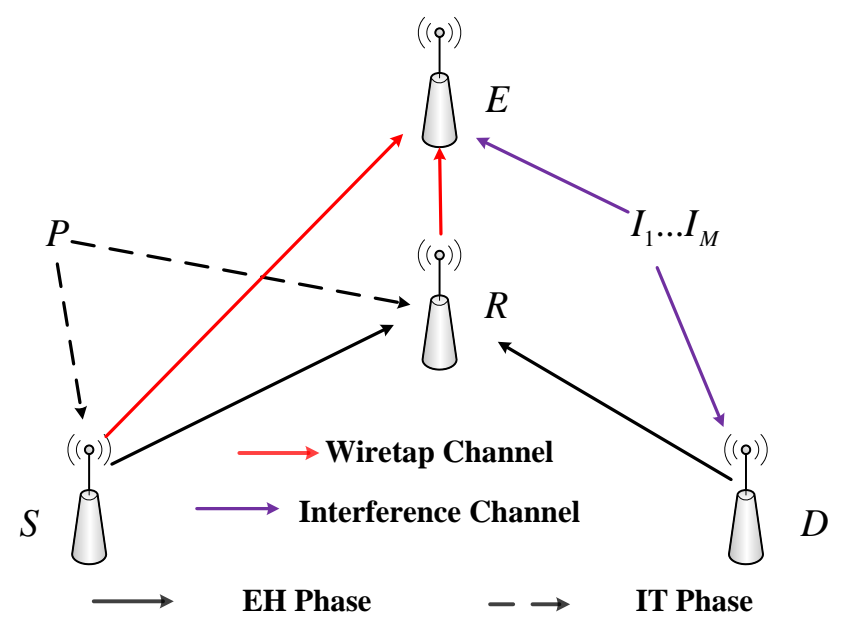

Figure 1. System model

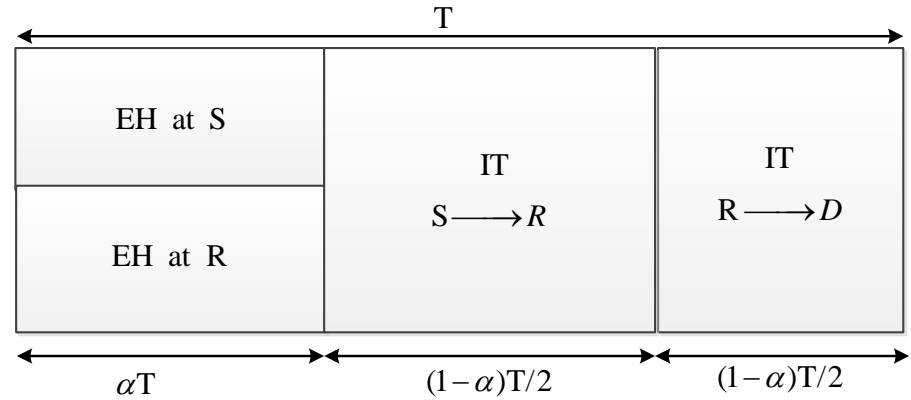

Figure 2. The EH and IT phases

\subsection{Energy harvesting phase}

In this phase, the source $\mathrm{S}$ and $\mathrm{R}$ will receive the power from the power beacon. Hence, the average transmit power at $\mathrm{S}$ and $\mathrm{R}$ can be formulated by, respectively:

$$
\begin{aligned}
& P_{S}=\frac{E_{S}}{(1-\alpha)(T / 2)}=\frac{2 \eta \alpha T P_{B}\left|h_{B S}\right|^{2}}{(1-\alpha) T}=\mu P_{B}\left|h_{B S}\right|^{2} \\
& P_{R}=\frac{E_{R}}{(1-\alpha)(T / 2)}=\frac{2 \eta \alpha T P_{B}\left|h_{B R}\right|^{2}}{(1-\alpha) T}=\mu P_{B}\left|h_{B R}\right|^{2}
\end{aligned}
$$

where $P_{B}$ is the transmit power at the power beacon $\mathrm{B}, \mu=\frac{2 \eta \alpha}{1-\alpha}$ and $0<\eta \leq 1$ : energy conversion efficiency.

\subsection{Information transmission phase}

In the second time slot, the $\mathrm{S}$ transmits its signal to $\mathrm{R}$, the corresponding received signal at the $\mathrm{R}$ can be expressed as,

$$
y_{R}=h_{S R} x_{S}+n_{R}
$$


where $x_{s}$ is the transmitted signal at the source $\mathrm{S}$ and satisfied $\mathrm{E}\left\{\left|x_{S}\right|^{2}\right\}=P_{S}$, wherein $\mathrm{E}\{\bullet\}$ is expectation operator and $n_{R}$ is the additive white Gaussian noise (AWGN) with variance $N_{0}$

In the third phase, the received signal at the destination $\mathrm{D}$ can be given by:

$$
y_{D}=h_{R D} x_{R}+\sum_{n=1}^{M} x_{I_{n}} h_{I_{n} D}+n_{D}
$$

where $x_{R}$ is the transmitted signal at the relay $\mathrm{R}$ and must be satisfied $\mathrm{E}\left\{\left|x_{R}\right|^{2}\right\}=P_{R}, \mathrm{n}_{\mathrm{D}}$ is the AWGN with variance $\mathrm{N}_{0}, h_{I_{n} D}$ is the channel gain between $\mathrm{n}^{\text {th }}$ interference source and destination.

To simplicity, we assume that all the interference sources have the same transmit power $P_{I}$, it means that $\frac{\mathrm{E}\left\{\left|x_{I_{n}}\right|^{2}\right\}}{n=1 \ldots M}=P_{I}$.

Next, the received signal at the Eavesdropper can be obtained by:

$$
y_{E}=h_{R E} x_{R}+h_{S E} x_{S}+\sum_{n=1}^{M} x_{I_{n}} h_{I_{n} E}+n_{E}
$$

where $h_{I_{n} E}$ is the channel gain between $\mathrm{n}^{\text {th }}$ interference source and eavesdropper and $\mathrm{n}_{\mathrm{E}}$ is also the AWGN with variance $\mathrm{N}_{0}$

From (3) and (4), the obtained signal-to-noise ratio (SNR) to successfully detect the data at R and D can be claimed as, respectively.

$$
\begin{aligned}
& \gamma_{R}=\frac{\left|h_{S R}\right|^{2} P_{S}}{N_{0}}, \\
& \gamma_{D}=\frac{\left|h_{R D}\right|^{2} P_{R}}{P_{I} \sum_{n=1}^{M}\left|h_{I_{n} D}\right|^{2}+N_{0}}
\end{aligned}
$$

By substituting (1) and (2) into (6), the (6) can be reformulated as

$$
\begin{aligned}
& \gamma_{R}=\mu \Psi\left|h_{S R}\right|^{2}\left|h_{B S}\right|^{2}=\mu \Psi X, \\
& \gamma_{D}=\frac{\mu \Psi\left|h_{R D}\right|^{2}\left|h_{B R}\right|^{2}}{\Delta \sum_{n=1}^{M}\left|h_{I_{n} D}\right|^{2}+1}=\frac{\mu \Psi Y}{\Delta Z+1}
\end{aligned}
$$

where $\Psi=\frac{P_{S}}{N_{0}}, \Delta=\frac{P_{I}}{N_{0}}, X=\left|h_{S R}\right|^{2}\left|h_{B S}\right|^{2}, Y=\left|h_{R D}\right|^{2}\left|h_{B R}\right|^{2}$ and $Z=\sum_{n=1}^{M}\left|h_{I_{n} D}\right|^{2}$

Similar to above, the obtained SNR at E can be given by

$$
\gamma_{E}=\frac{\mu \Psi\left|h_{R E}\right|^{2}\left|h_{B R}\right|^{2}+\mu \Psi\left|h_{S E}\right|^{2}\left|h_{B S}\right|^{2}}{\Delta \sum_{n=1}^{M}\left|h_{I_{n} E}\right|^{2}+1}=\frac{\mu \Psi(T+U)}{\Delta Q+1}
$$

where $T=\left|h_{R E}\right|^{2}\left|h_{B R}\right|^{2}, U=\left|h_{S E}\right|^{2}\left|h_{B S}\right|^{2}$ and $Q=\sum_{n=1}^{M}\left|h_{I_{n} E}\right|^{2}$

\section{Remark 1:}


In [2], the probability density function (PDF) of the random variable (RV) $\mathrm{Z}$ and $\mathrm{Q}$ can be obtained as, respectively.

$$
\begin{aligned}
& f_{Z}(t)=\frac{\left(\lambda_{I D}\right)^{M}}{(M-1) !} t^{M-1} \exp \left(-\lambda_{I D} t\right), \\
& f_{Q}(t)=\frac{\left(\lambda_{I E}\right)^{M}}{(M-1) !} t^{M-1} \exp \left(-\lambda_{I E} t\right)
\end{aligned}
$$

Where $\lambda_{I D}$ and $\lambda_{I E}$ are the mean of RV Z and Q, respectively.

\section{SYSTEM PERFORMANCE ANALYSIS}

\subsection{Outage probability (OP)}

The OP of the system can be computed as,

$$
\begin{aligned}
O P & =\operatorname{Pr}\left(\min \left(\gamma_{R}, \gamma_{D}\right)<\gamma_{t h}\right)=\operatorname{Pr}\left[\min \left(\mu \Psi X, \frac{\mu \Psi Y}{\Delta Z+1}\right)<\gamma_{t h}\right] \\
& =1-\underbrace{\operatorname{Pr}\left(\mu \Psi X \geq \gamma_{t h}\right)}_{P_{1}} \times \underbrace{\operatorname{Pr}\left(\frac{\mu \Psi Y}{\Delta Z+1} \geq \gamma_{t h}\right)}_{P_{2}}
\end{aligned}
$$

where $\gamma_{t h}=2^{2 R}-1$ is the threshold of the system, and $\mathrm{R}$ is target rate.

From (10), $\mathrm{P}_{1}$ can be calculated by:

$$
\begin{aligned}
& P_{1}=1-\operatorname{Pr}\left(\mu \Psi X<\gamma_{t h}\right)=1-\operatorname{Pr}\left(\left|h_{S R}\right|^{2}\left|h_{B S}\right|^{2}<\frac{\gamma_{t h}}{\mu \Psi}\right) \\
& =1-\int_{0}^{\infty} F_{\left|h_{S R}\right|^{2}}\left(\frac{\gamma_{t h}}{\mu \Psi x}\right) \times f_{\left|h_{B S}\right|^{2}}(x) d x \\
& =1-\int_{0}^{\infty} \lambda_{B S}\left(1-\exp \left(-\frac{\lambda_{S R} \gamma_{t h}}{\mu \Psi x}\right)\right) \times \exp \left(-\lambda_{B S} x\right) d x \\
& =\int_{0}^{\infty} \lambda_{B S} \exp \left(-\frac{\lambda_{S R} \gamma_{t h}}{\mu \Psi x}-\lambda_{B S} x\right) d x
\end{aligned}
$$

where $\lambda_{S R}$ and $\lambda_{B S}$ are the mean of RV $\left|h_{S R}\right|^{2}$ and $\left|h_{B S}\right|^{2}$,

Here, the euation (11) can be rewritten as,

$$
P_{1}=2 \sqrt{\frac{\lambda_{S R} \lambda_{B S} \gamma_{t h}}{\mu \Psi}} \times K_{1}\left(2 \sqrt{\frac{\lambda_{S R} \lambda_{B S} \gamma_{t h}}{\mu \Psi}}\right)
$$

where $K_{v}(\bullet)$ is the modified Bessel function of the second kind and $\mathrm{v}^{\text {th }}$ order.

Next, $\mathrm{P}_{2}$ can be derived by:

$$
P_{2}=1-\operatorname{Pr}\left(\frac{\tilde{Y}}{\Delta Z+1}<\gamma_{t h}\right)=1-\int_{0}^{\infty} F_{\tilde{Y}}\left(\gamma_{t h}(\Delta t+1)\right) \times f_{Z}(t) d t
$$

where $\tilde{Y}=\mu \Psi Y$.

We apply the result from (12) and then substitute (9) into (13), $\mathrm{P}_{2}$ can be obtained as,

$$
P_{2}=\frac{2\left(\lambda_{I D}\right)^{M}}{(M-1) !} \int_{0}^{\infty} t^{M-1} \exp \left(-\lambda_{I D} t\right) \times \sqrt{\frac{\lambda_{R D} \lambda_{B R} \gamma_{t h}(\Delta t+1)}{\mu \Psi}} \times K_{1}\left(2 \sqrt{\frac{\lambda_{R D} \lambda_{B R} \gamma_{t h}(\Delta t+1)}{\mu \Psi}}\right) d t
$$


Finally, substituting (12) and (14) into (10), the OP in the final form as,

$$
\begin{aligned}
& O P=1-\frac{4\left(\lambda_{I D}\right)^{M}}{(M-1) !} \sqrt{\frac{\lambda_{S R} \lambda_{B S} \gamma_{t h}}{\mu \Psi}} \times K_{1}\left(2 \sqrt{\frac{\lambda_{S R} \lambda_{B S} \gamma_{t h}}{\mu \Psi}}\right) \\
& \times \int_{0}^{\infty} t^{M-1} \exp \left(-\lambda_{I D} t\right) \times \sqrt{\frac{\lambda_{R D} \lambda_{B R} \gamma_{t h}(\Delta t+1)}{\mu \Psi}} \times K_{1}\left(2 \sqrt{\frac{\lambda_{R D} \lambda_{B R} \gamma_{t h}(\Delta t+1)}{\mu \Psi}}\right) d t
\end{aligned}
$$

\subsection{Intercept probability (IP)}

The IP can be defined as,

$$
\begin{aligned}
& I P=\operatorname{Pr}\left(\gamma_{E} \geq \gamma_{t h}\right)=1-\operatorname{Pr}\left(\gamma_{E}<\gamma_{t h}\right) \\
& =1-\operatorname{Pr}\left(\frac{\mu \Psi(T+U)}{\Delta Q+1}<\gamma_{t h}\right)=1-\int_{0}^{\infty} F_{\Sigma}\left(\gamma_{t h}(\Delta x+1)\right) \times f_{Q}(x) d x
\end{aligned}
$$

where $\Sigma=\mu \Psi(T+U)$

The CDF of $\Sigma$ can be found as,

$$
\begin{aligned}
& F_{\Sigma}(y)=\operatorname{Pr}(\Sigma<y)=\operatorname{Pr}(\mu \Psi(T+U)<y) \\
& =\operatorname{Pr}\left(T+U<\frac{y}{\mu \Psi}\right)=\int_{0}^{\frac{y}{\mu \Psi}} F_{U}\left(\frac{y}{\mu \Psi}-t\right) \times f_{T}(t) d t
\end{aligned}
$$

By using the result from (12) and formula $\frac{d}{d x}\left(x^{v} K_{v}(x)\right)=-x^{v} K_{v-1}(x)$, the PDF of $\mathrm{T}$ can be computed by:

$$
f_{T}(t)=2 \lambda_{R E} \lambda_{B R} \times K_{0}\left(2 \sqrt{\lambda_{R E} \lambda_{B R} t}\right)
$$

where $\lambda_{R E}$ and $\lambda_{B R}$ are the mean of RV $\left|h_{R E}\right|^{2}$ and $\left|h_{B R}\right|^{2}$, respectively.

$$
\text { And } F_{U}\left(\frac{y}{\mu \Psi}-t\right)=\left\{1-2 \sqrt{\lambda_{S E} \lambda_{B S}\left(\frac{y}{\mu \Psi}-t\right)} \times K_{1}\left(2 \sqrt{\lambda_{S E} \lambda_{B S}\left(\frac{y}{\mu \Psi}-t\right)}\right)\right\}
$$

where $\lambda_{S E}$ and $\lambda_{B S}$ are the mean of RV $\left|h_{S E}\right|^{2}$ and $\left|h_{B S}\right|^{2}$, respectively.

Substituting (18), (19) into (17), and then into (16), the IP can be claimed by:

$$
\begin{aligned}
& I P=1-2 \lambda_{R E} \lambda_{B R} \int_{0}^{\infty} \int_{0}^{\frac{\gamma_{t h}(\Delta x+1)}{\mu \Psi}}\left\{1-2 \sqrt{\lambda_{S E} \lambda_{B S}\left(\frac{\gamma_{t h}(\Delta x+1)}{\mu \Psi}-t\right)} \times K_{1}\left(2 \sqrt{\lambda_{S E} \lambda_{B S}\left(\frac{\gamma_{t h}(\Delta x+1)}{\mu \Psi}-t\right)}\right)\right\} \\
& \times \frac{\left(\lambda_{I E}\right)^{M}}{(M-1) !} x^{M-1} \exp \left(-\lambda_{I E} x\right) \times K_{0}\left(2 \sqrt{\lambda_{R E} \lambda_{B R} t}\right) d t d x
\end{aligned}
$$

\section{NUMERICAL RESULTS AND DISCUSSION}

Figure 4 draws the impact of $\psi$ on the system OP with the main system parameters as $\eta=0.8, \alpha=0.5$, and $\Delta=1 \mathrm{~dB}$, respectively. The Figure shows that the OP falls in the rising direction of $\psi$. Furthermore, the IP 
is considered as the function of $\psi$, as shown in Figure 4. As shown in Figure 4, IP increases when $\psi$ rises. From Figures 3 and 4, we can see that the analytical and the simulation curves are the same as the analytical section.

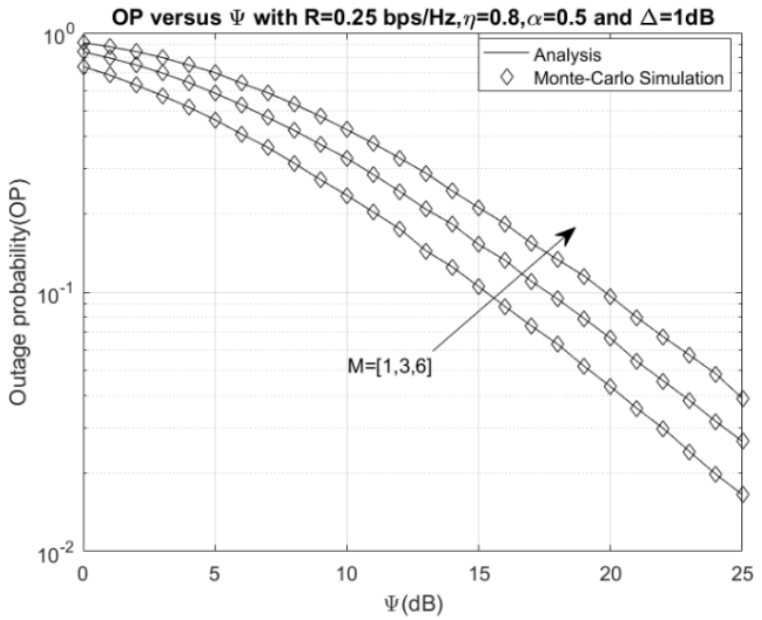

Figure 3. OP versus $\psi$

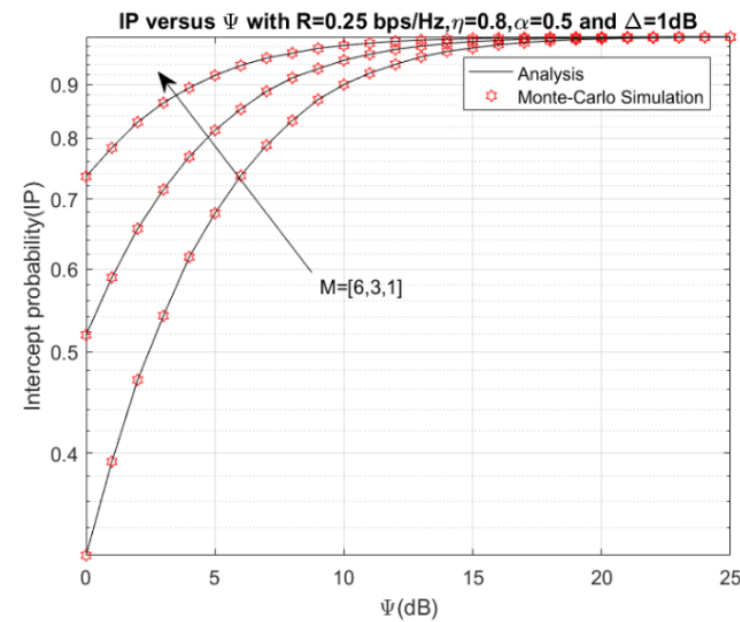

Figure 4. IP versus $\psi$

Moreover, the system OP and IP versus $\alpha$ are considered in Figures 5 and 6, respectively. In these Figures, we set $\eta=0.8, \beta=0.5$, and $\psi=2 \mathrm{~dB}$. From Figures 5 and 6 , we can state that the system OP has a massive increase and IP decreases with rising $\alpha$, respectively. In addition, the simulation and analytical values are the same.

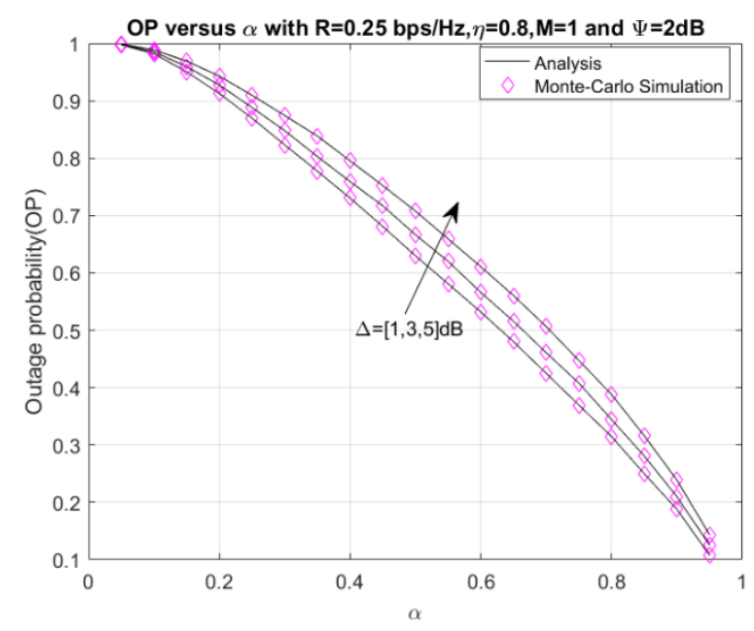

Figure 5. OP versus $\alpha$

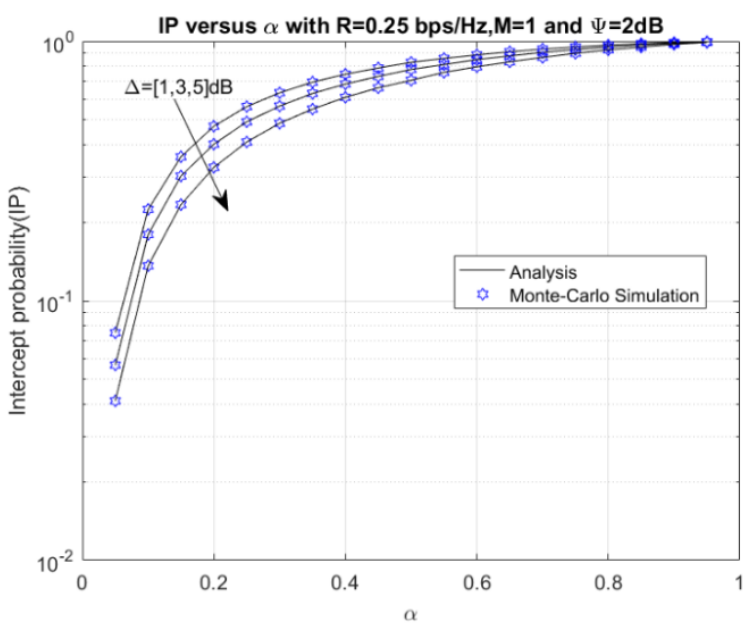

Figure 6. IP versus $\alpha$.

Finally, the system OP and IP versus $\mathrm{M}$ are considered in Figures 7 and 8, respectively. In these Figures, we set $\eta=0.8, \beta=0.5$, and $\psi=2 \mathrm{~dB}$. From Figures 7 and 8 , we can see that the system OP has a massive increase and IP decreases with rising $\mathrm{M}$, respectively. And the simulatiton ovelaps the analystical values. 


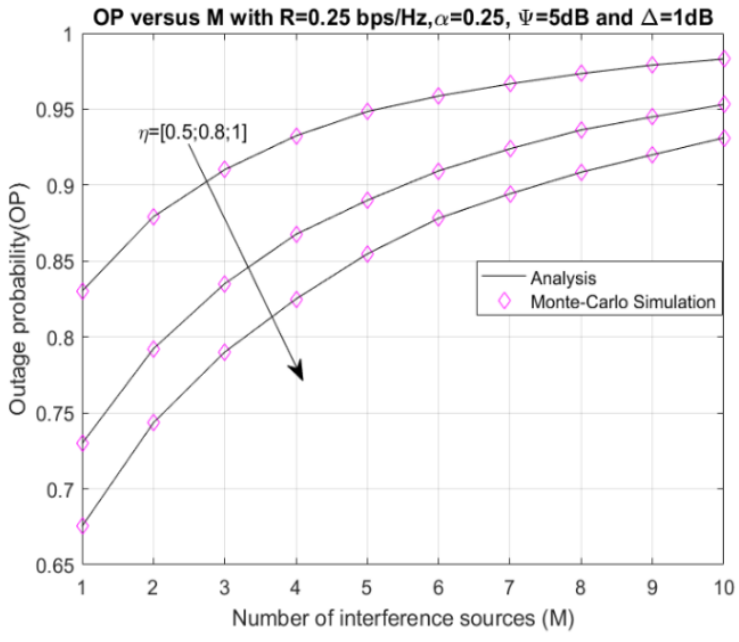

Figure 7. OP versus M

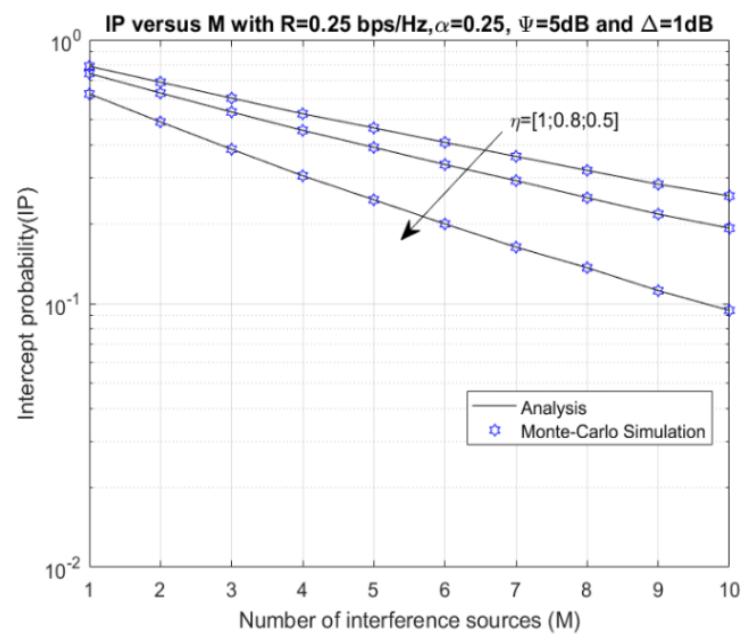

Figure 8. IP versus M

\section{CONCLUSION}

Performance analysis of power beacon-assisted D2D communication networks in the presence of eavesdropper and co-channel interference is presented is investigated. The outage probability and the intercept probability of the proposed system are analyzed and derived. The impact of the main system parameters on the system performance is investigated. The monte carlo simulation is used for verifying the correctness of the analytical section.

\section{REFERENCES}

[1] Bi, S., Ho, C.K., and Zhang, R., "Wireless powered communication: Opportunities and challenges," IEEE Commun. Mag, vol. 53, no. 4, pp. 117-125, 2015, doi: 10.1109/mcom.2015.7081084.

[2] Niyato, D., Kim, D.I., Maso, M., and Han, Z., "Wireless Powered Communication Networks: Research Directions and Technological Approaches," IEEE Wirel. Commun, pp. 2-11, 2017, doi: 10.1109/mwc.2017.1600116.

[3] Yu, H., Lee, H., and Jeon, H., "What is 5G? Emerging 5G Mobile Services and Network Requirements," Sustainability, vol. 9, no. 10, p. 1848, 2017, doi: 10.3390/su9101848.

[4] Zhou, X., Zhang, R., and Ho, C.K., "Wireless Information and Power Transfer: Architecture Design and RateEnergy Tradeoff," IEEE Trans. Commun, vol. 61, no. 11, pp. 4754-4767, 2013, doi: 10.1109/tcomm.2013.13.120855.

[5] Nguyen, Tan. N., Tran M., Ha D-H., Trang T. T., and Voznak M.. "Multi-source in DF Cooperative Networks with the PSR Protocol Based Full-duplex Energy Harvesting over a Rayleigh Fading Channel: Performance Analysis," Proceedings of the Estonian Academy of Sciences, vol. 68, 2019, p. 264, doi: 10.3176/proc.2019.3.03.

[6] Nguyen, Tan N., Minh Tran., Thanh-Long Nguyen., Duy-Hung Ha., and Miroslav Voznak, "Performance Analysis of a User Selection Protocol in Cooperative Networks with Power Splitting Protocol-Based Energy Harvesting Over Nakagami-m/Rayleigh Channels," Electronics vol. 8, p. 448, 2019, doi: 10.3390/electronics8040448.

[7] Nguyen, Tan N., Quang Minh, T., Tran, P., and Vozňák, M., "Energy Harvesting over Rician Fading Channel: A Performance Analysis for Half-Duplex Bidirectional Sensor Networks under Hardware Impairments," Sensors, vol. 18, no. 6, p. 1781, 2018, doi: 10.3390/s18061781.

[8] Nguyen, Tan N et al., "Performance Enhancement for Energy Harvesting Based Two-way Relay Protocols in Wireless Ad-hoc Networks with Partial and Full Relay Selection Methods," Ad Hoc Networks, vol. 84, p. 178-187, 2019, doi: 10.1016/j.adhoc.2018.10.005.

[9] Tin, Dinh Nguyen, and Ha, Trang, "power beacon assisted Energy Harvesting Wireless Physical Layer Cooperative Relaying Networks: Performance Analysis," Symmetry, 2020, doi: 10.3390/sym12010106.

[10] Gopala P. K., Lai L., and El Gamal H., "On the Secrecy Capacity of Fading Channels," in IEEE Transactions on Information Theory, vol. 54, no. 10, pp. 4687-4698, 2008, doi: 10.1109/TIT.2008.928990.

[11] Sun, Li., and Qinghe Du., "A Review of Physical Layer Security Techniques for Internet of Things: Challenges and Solutions," Entropy, vol. 20, no. 10, p. 730, 2018, doi: 10.3390/e20100730.

[12] Kuhestani, Ali, Abbas Mohammadi, and Mohammadali Mohammadi, "Joint Relay Selection and Power Allocation in Large-Scale MIMO Systems with Untrusted Relays and Passive Eavesdroppers," IEEE Transactions on Information Forensics and Security, vol. 13, no. 2, pp. 341-355, 2017, doi: 10.1109/tifs.2017.2750102.

[13] Hu, Lin et al., "Cooperative Jamming for Physical Layer Security Enhancement in Internet of Things," IEEE Internet of Things Journal, vol. 5, no. 1, pp. 219-28, 2018, doi: 10.1109/jiot.2017.2778185. 
[14] Tin, Phu Tran., Dang The Hung., Nguyen, Tan N., Tran Duy, and Miroslav Voznak., "Secrecy Performance Enhancement for Underlay Cognitive Radio Networks Employing Cooperative Multi-Hop Transmission with and without Presence of Hardware Impairments," Entropy, vol. 21, no. 2, p. 217, 2019, doi: 10.3390/e21020217.

[15] Zhao, R., Yuan, Y., Fan, L., and He, Y.-C, "Secrecy Performance Analysis of Cognitive Decode-and-Forward Relay Networks in Nakagami-m Fading Channels," IEEE Trans. Commun, vol. 65, no. 2, pp. 549-563, 2017, doi: 10.1109/TCOMM.2016.2618793.

[16] Phu Tran Tin., Minh Tran, Nguyen, Tan N,, and Thanh-Long Nguyen, "System performance analysis of hybrid timepower switching protocol of EH bidirectional relaying network in amplify-and-forward mode," Indonesian Journal of Electrical Engineering and Computer Science, vol. 14, no. 1, pp. 118-126, 2019, doi: 10.11591/ijeecs.v14.i1.pp123-131.

[17] Phu Tran Tin,, Minh Tran,, Nguyen, Tan N,, and Thanh-Long Nguyen,, "A new look at energy harvesting halfduplex DF power splitting protocol relay network over rician channel in case of maximizing capacity," Indonesian Journal of Electrical Engineering and Computer Science, vol. 13, no. 1, pp. 249-257, 2019, doi: 10.11591/ijeecs.v13.i1.pp249-257.

[18] Nguyen, Tan N et al., "Throughput Enhancement in FD-and SWIPT-enabled IoT Networks over Non-Identical Rayleigh Fading Channels," in IEEE Internet of Things Journal, doi: 10.1109/JIOT.2021.3120766.

[19] Nguyen, B C et al., "Cooperative Communications for Improving the Performance of Bidirectional Full-Duplex System With Multiple Reconfigurable Intelligent Surfaces," in IEEE Access, vol. 9, pp. 134733-134742, 2021, doi: 10.1109/ACCESS.2021.3114713.

[20] Van-Duc Phan., Phu Tran Tin., Minh Tran., and Tran Thanh Trang., "User selection protocols in FD PSP EH cooperative network over rayleigh fading channel: outage and intercept probability," International Journal of Power Electronics and Drive Systems (IJPEDS), vol. 10, no. 4, p. 2130, 2019, doi: 10.11591/ijpeds.v10.i4.pp2130-2137.

[21] Ju, H., and Zhang, R., "Throughput Maximization in Wireless Powered Communication Networks," IEEE Trans. Wirel. Commun, vol. 13, no. 1, pp. 418-428, 2014, doi: 10.1109/TWC.2013.112513.130760.

[22] Bhatnagar, M.R., "On the Capacity of Decode-and-Forward Relaying over Rician Fading Channels," IEEE Commun. Lett, vol. 17, no. 6, pp. 1100-1103, 2013, doi: 10.1109/lcomm.2013.050313.122813.

[23] Daniel Zwillinger," "Table of Integrals, Series, and Products," Springer: NY, USA, 2015, doi: 10.1016/c2010-064839-5.

[24] Nasir, A.A., Zhou, X., Durrani, S., and Kennedy, R. A., "Relaying Protocols for Wireless Energy Harvesting and Information Processing," IEEE Trans. Wirel. Commun, vol. 12, no. 7, pp. 3622-3636, 2013, doi: 10.1109/twc.2013.062413.122042.

[25] Nguyen, Tan N., T.H.Q., Minh, P., Tran, T., and Voznak, M., “Adaptive Energy Harvesting Relaying Protocol for Two-Way Half Duplex System Network over Rician Fading Channels,” Wirel. Commun. Mob. Comput, 2018, doi: 10.1155/2018/7693016. 